\title{
Aberrant regulation of RNA splicing in sunflower hybrids may underlie intrinsic incompatibilities
}

\section{Short title}

Transgressive splicing in sunflowers

Chris C R Smith ${ }^{1}$, Loren H Rieseberg ${ }^{2}$, Brent S Hulke ${ }^{3}$, and Nolan C Kane ${ }^{1}$

${ }^{1}$ Department of Ecology and Evolutionary Biology, University of Colorado, Boulder; 2Department of Botany, University of British Columbia, Vancouver, BC, Canada; 3 USDA-ARS Edward T Schafer Agricultural Research Center, Fargo, ND, USA

\author{
${ }^{*}$ Corresponding author \\ Chris C R Smith \\ BioFrontiers Institute \\ 3415 Colorado Ave \\ 596 UCB \\ Boulder, CO 80308-0596, USA \\ E-mail: chriscs@colorado.edu
}




\section{Abstract}

2 Alternative spicing is an integral part of gene expression in multicellular organisms that

3 allows for diverse mRNA transcripts and proteins to be produced from a single gene.

4 However, most existing analyses have focused on macro-evolution, with only limited

5 research on splice site evolution over shorter term, micro-evolutionary time scales. Here

6 we examine splicing evolution that has occurred during domestication and observe 45

7 novel splice forms with strongly transgressive isoform compositions, representing $0.24 \%$

8 of analyzed transcripts. We identify loci associated with variation in the levels of these

9 splice forms, finding that many novel transcripts were regulated by multiple alleles with

10 non-additive interactions. A subset of these interactions involved the expression of

11 individual spliceosome components. These overdominant and epistatic interactions

12 often resulted in alteration in the protein-coding regions of the transcripts, resulting in

13 frameshifts and truncations. By associating the splice variation in these genes with size

14 and growth rate measurements, we found that none of the individual splice variants

15 affected these plant traits significantly, but the cumulative expression of all aberrant

16 transcripts did show a significant reduction in growth rate associated with higher

17 proportions of disrupted transcripts. This demonstrates the importance of co-evolution of

18 the different spliceosomal components and their regulators and suggests that these

19 genes may contribute to evolution of reproductive isolation as Bateson-Dobzhansky-

20 Muller incompatibility loci. 
23 In multicellular organisms, it is common that segments of pre-mRNA molecules are

24 physically removed, and the remaining segments are spliced back together. Through

25 splicing alternative combinations of segments together, organisms produce various

26 mRNA molecules, and thus multiple proteins, using the information encoded in a single

27 gene. Here, we investigated the RNA of two sunflower genotypes, one wild and one

28 domesticated, as well as the hybrid offspring resulting from a cross between the two

29 genotypes. We found certain mRNA molecules that were spliced exclusively in the

30 hybrids and were absent in the examined parental lines. These unique hybrid mRNAs

31 were predicted to be consequential for the hybrids' health, and thus represented a

32 malfunction in the mechanisms that regulate splicing. These results improve our

33 understanding of the genetic regulation of alternative splicing and how alternative splice

34 forms evolve. Our findings may lead to further inquiries about how aberrant splicing

35 promotes the formation of new species in nature.

\section{Introduction}

38 Gene expression in multicellular organisms involves alternative splicing, which uses the

39 information encoded in a single gene to create an assortment of mRNA transcripts. For

40 example, as many as 150,000 unique transcripts were found in Arabidopsis thaliana

41 (Huang et al., 2019a), a plant with only 27,466 protein coding genes (Cheng et al.,

42 2017). Furthermore, relatively few splicing isoforms are conserved among plant species:

43 only $14.7 \%$ of isoforms are conserved between Arabidopsis and rice, and only $16.4 \%$

44 are conserved between rice and maize (Severing et al., 2009; Zhang et al., 2015). 
45 Despite the large diversity of isoforms found in nature, our knowledge about the

46 evolution of splicing, and the origins of novel splice types, is limited.

47 Recent studies have shown that RNA splicing may change over relatively short

48 evolutionary timescales, e.g. 1,000-10,000 years (Thatcher et al., 2014; Zhang and Xiao

49 2018; Smith et al., 2018; Khokhar et al., 2019; Huang et al., 2019b; Lin et al., 2020). In

50 domesticated sunflowers, divergent splice forms were identified that seemed to have

51 arisen from standing variation (Smith et al., 2018). In this case, the divergent splice

52 forms found in domesticated genotypes were also sometimes found in wild populations,

53 suggesting that this variation existed at some frequency in the ancestral population

54 before drift or selection brought certain splice forms to fixation in domesticated cultivars.

55 Several questions remain: In hybrids, how often is splicing transgressive, leading to

56 variation not seen in parental lineages? Are novel transcripts costly in hybrids? At a

57 genetic level, what regulation appears to be associated with novel splicing?

58 Hybridization is one possible source of phenotypic variation in this case (Lewontin and

59 Birch, 1966; Scascitelli et al., 2010). In regard to RNA splicing, a hybrid individual will

60 inherit regulatory alleles from each parent and thus could create a set of transcripts that

61 resembles that of either or both parents. On the other hand, it is possible that new

62 alleles that were benign in one parent genetic background may interact with unfamiliar

63 alleles in the opposite parent genome (Bateson, 1909; Dobzhansky, 1936; Muller,

64 1942), causing mis-regulation of splicing and the formation of novel RNA transcripts

65 (Fig. 1). 


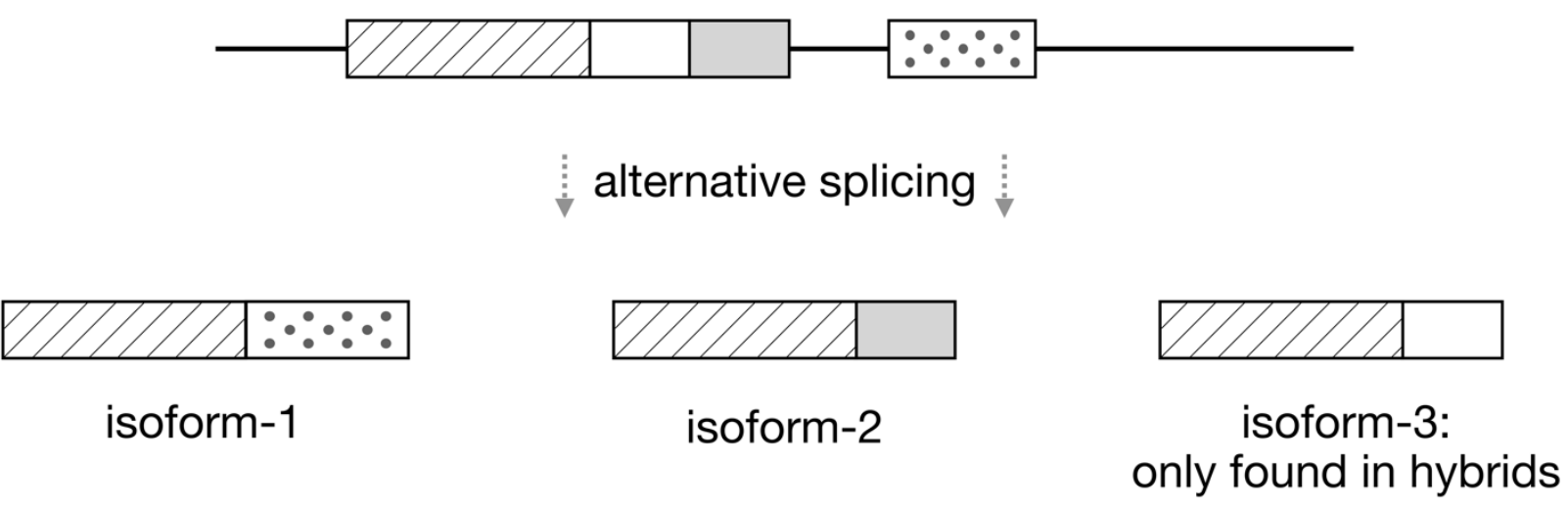

67 Figure 1. Alternative splicing diagram. The black line shows a DNA strand, and shaded boxes represent exon or retained intron segments. The bottom boxes are mature mRNA transcripts resulting from alternative splicing. We refer to theoretical isoform-3 as a novel, transgressive isoform because it is found exclusively in hybrids. Other isoforms could be transgressive if spliced at extreme levels relative to the parent taxa.

72 This phenomenon may be thought of as an example of transgressive segregation,

73 because the hybrid phenotype is more extreme than- or in this case, nonexistent in-

74 either parent (Slatkin and Lande, 1994). Transgressive segregation is not uncommon

75 for a wide range of phenotypes, with $36-44 \%$ of examined traits in hybridizing systems

76 showing some degree of transgression (Rieseberg et al., 1999; Stelkens and

77 Seehauson, 2009). Transgressive segregation has also been observed in splice isoform

78 variation (Scascitelli et al., 2010), although these observations have been limited to

79 relatively few isoforms and the genetic regulatory mechanisms have not been identified.

80 In general, the predominant genetic basis for transgression is complementary gene

81 action, meaning that some proportion of hybrids may have alleles from each parent that

82 combine additively to produce the extreme phenotype (see the first table from 
83 Rieseberg et al., 1999). Other causes of transgressive segregation include interactions

84 between alleles at different loci, i.e. epistasis, or within a locus, i.e. overdominance.

85 Here, we investigated transgressive splice forms in the common sunflower, Helianthus

86 annuus. We surveyed the entire transcriptome of one hundred recombinant inbred lines

87 (RILs) derived from a wild $\mathrm{x}$ domesticated cross and mapped the genetic basis of

88 transgressive splicing. Our findings help us understand how alternative splice forms

89 evolve and how genetic incompatibilities contribute to the formation of new species.

\section{Results}

92 Characterization of novel splice forms in hybrids

93 We identified 45 isoforms that were spliced in at least one hybrid at a substantial level (

$94 \geq 1$ transcripts per million; TPM), were absent in all six parent samples (zero reads),

95 and appeared to each be an alternative splice form of a corresponding parent isoform

96 (Table 1). We refer to these as "novel" isoforms to describe that they were not detected

97 in the parent samples. These 45 isoforms represented $0.24 \%$ of analyzed transcripts

98 after initial filtering. All exons and retained intron sequences in the novel transcripts

99 were present in the Ha412-HO genome; this check supports that these transcripts

100 represent alternative splice forms instead of sequence mutations, e.g. indels. Additional

101 cases of transgressive splicing potentially exist, but these represented the most

102 convincing examples in the current dataset. 


\begin{tabular}{|c|c|c|c|c|c|c|c|}
\hline ID & $\begin{array}{l}\text { Chrom., } \\
\mathrm{cM}\end{array}$ & $\begin{array}{l}\text { Length } \\
\text { (bp) }\end{array}$ & Annotation & $\begin{array}{l}\text { Splice } \\
\text { type }\end{array}$ & $\begin{array}{l}\text { OR } \\
F\end{array}$ & $\begin{array}{l}\text { Genetic } \\
\text { basis }\end{array}$ & Spliceosome component associations \\
\hline 1 & $12,63.99$ & 632 & cytochrome B561-1 & IR,ES,AS & $E$ & & \\
\hline 2 & $3,24.64$ & 766 & $\begin{array}{l}\text { sucrose phosphate synthase } \\
2 \mathrm{~F}\end{array}$ & IR,ES,AS & $\mathrm{T}$ & $E$ & RNA helicase DDX23/PRP28 \\
\hline 3 & $16,42.46$ & 1,231 & & IR,ES,AS & $\mathrm{T}$ & $\mathrm{O}$ & \\
\hline 4 & $17,47.71$ & 2,773 & $\begin{array}{l}\text { ATPase E1-E2 type family } \\
\text { protein / haloacid } \\
\text { dehalogenase-like hydrolase }\end{array}$ & IR,ES,AS & $E$ & & \\
\hline 5 & $17,47.71$ & 2,868 & $\begin{array}{l}\text { ATPase E1-E2 type family } \\
\text { protein / haloacid } \\
\text { dehalogenase-like hydrolase }\end{array}$ & IR,ES & $S$ & $E$ & \\
\hline 6 & $6,29.29$ & 1,638 & $\begin{array}{l}\text { glycerol-3-phosphate } \\
\text { acyltransferase }\end{array}$ & IR,ES,AS & $S$ & $E$ & $\begin{array}{l}\text { pre-mRNA-splicing factor } \\
\text { RBM22/SLT11, U6 snRNA-associated } \\
\text { Sm-like protein LSm6/LSm7 }\end{array}$ \\
\hline 7 & $17,69.49$ & 1,251 & $\begin{array}{l}\text { UBX domain-containing } \\
\text { protein }\end{array}$ & IR,ES & $\mathrm{T}$ & & \\
\hline 8 & $5,51.16$ & 1,771 & $\begin{array}{l}\mathrm{FAD} / \mathrm{NAD}(\mathrm{P}) \text {-binding } \\
\text { oxidoreductase }\end{array}$ & IR,ES,AS & $\mathrm{T}$ & $E$ & \\
\hline 9 & $12,64.01$ & 3,162 & ABC-2 type transporter & IR,ES & $\mathrm{T}$ & $E$ & $\begin{array}{l}\text { ATP-dependent RNA helicase } \\
\text { DDX23/PRP28 1, nuclear cap-binding } \\
\text { protein, pre-mRNA-splicing helicase } \\
\text { BRR2, U5 small nuclear } \\
\text { ribonucleoprotein helicase }\end{array}$ \\
\hline 10 & $12,64.01$ & 3,274 & ABC-2 type transporter & IR,ES,AS & $\mathrm{T}$ & $E$ & $\begin{array}{l}\text { U6 snRNA-associated Sm-like protein } \\
\text { LSm6 }\end{array}$ \\
\hline 11 & $8,67.05$ & 2,701 & $\begin{array}{l}\text { Mitochondrial transcription } \\
\text { termination factor }\end{array}$ & IR,ES,AS & $E$ & $\mathrm{O}$ & $\begin{array}{l}\text { U4/U6 small nuclear ribonucleoprotein } \\
\text { PRP31, small nuclear ribonucleoprotein } \\
\text { G }\end{array}$ \\
\hline 12 & $4,57.32$ & 2,441 & $\begin{array}{l}\text { Insulinase (Peptidase family } \\
\text { M16) }\end{array}$ & IR,ES,AS & $\mathrm{T}$ & $\mathrm{O}$ & small nuclear ribonucleoprotein D2 \\
\hline 13 & $7,10.48$ & 672 & indeterminate(ID)-domain 2 & IR,ES & $\mathrm{T}$ & & \\
\hline 14 & $16,50.99$ & 633 & $\begin{array}{l}\text { vascular plant one zinc finger } \\
\text { protein }\end{array}$ & IR,ES,AS & $S$ & (qe) & \\
\hline 15 & $14,25.78$ & 1,259 & $\begin{array}{l}\mathrm{NAD}(\mathrm{P}) \mathrm{H} \text { dehydrogenase } \\
\text { (quinone)s }\end{array}$ & IR,ES,AS & $\mathrm{T}$ & & \\
\hline 16 & $15,57.81$ & 1,309 & $\begin{array}{l}\text { P-loop containing nucleoside } \\
\text { triphosphate hydrolase }\end{array}$ & IR & $\mathrm{T}$ & $E$ & U4 \\
\hline 17 & $14,2.821$ & 4,783 & P-glycoprotein 13 & IR & $\mathrm{T}$ & $E$ & \\
\hline 18 & $4,50.94$ & 1,197 & $\begin{array}{l}\text { Leucine-rich repeat protein } \\
\text { kinase }\end{array}$ & IR,AS & $\mathrm{T}$ & & \\
\hline 19 & $11,59.05$ & 604 & & IR,ES, & $E$ & EOS & PRP38 family protein \\
\hline 20 & $11,44.40$ & 1,536 & Aldolase superfamily protein & IR,ES,AS & $\mathrm{E}$ & $E$ & PRP38 family protein \\
\hline 21 & $2,80.65$ & 368 & & IR,ES & $S$ & (q) & \\
\hline 22 & 7,11.01 & 1,557 & S-locus lectin protein kinase & IR,ES & S & & \\
\hline
\end{tabular}




\begin{tabular}{l|lll}
23 & $9,36.52$ & 1,379 & RNI-like superfamily protein \\
24 & $5,56.84$ & 2,338 & ARM repeat superfamily \\
25 & $2,32.95$ & 1,641 & Ikzf5 (DUF668)
\end{tabular}

UDP-N-acetylglucosamine

(UAA) transporter family

Uncharacterized protein

family (UPF0016)

auxin F-box protein 5

oligopeptide transporter 7

FkbM family

methyltransferase

FkbM family

methyltransferase

alanine:glyoxylate

aminotransferase

alanine:glyoxylate

aminotransferase

alanine:glyoxylate

aminotransferase

Inositol monophosphatase

family protein

$9,24.77 \quad 2,213$

Ribosomal protein $\mathrm{S} 18$

membrane protein

Terpenoid cyclases/Protein

prenyltransferases

sister-chromatid cohesion

protein 3

D-aminoacid

$\begin{array}{ccc}E S, A S & T & (q) \\ \text { IR } & T & O\end{array}$

IR,ES T

IR,ES,AS E

IR,ES,AS T

IR,ES,AS S

IR,other $S$

ES,AS S

IR,ES T

7,9.847 2,173 aminotransferase-like PLPdependent enzymes

105 The length of novel transcripts ranged from 368 to $4,783 \mathrm{bp}$ (median $=1,431 \mathrm{bp}$ ),

106 which was longer than the most similar parent isoform in $96 \%$ of cases. The intron and

107 exon sequences that differed between each transgressive isoform and the

108 corresponding parent isoform ranged from 20 to $1,741 \mathrm{bp}$ (median $=105 \mathrm{bp}$ ). The

109 number of RILs with $\geq 1$ TPM of each transgressive isoform ranged from 1 to 60

110 (median $=3)$. The proportion of alternative splicing composition at the gene-level was 
111 usually low for each novel isoform, although five were spliced at higher proportions ( >

$11260 \%)$. In other words, the transgressive isoform was usually a minor isoform while the

113 parent isoform remained the dominant transcript, but sometimes the novel isoform was

114 spliced as the dominant isoform or as the only isoform. Some hybrids were more

115 transgressive than others: all examined hybrids had some splicing of at least five

116 different transgressive isoforms, but some had up to 18 different transgressive isoforms.

117 The transgressive genes had 1 to 11 isoforms in the parent samples (median $=4$ ),

118 suggesting that genes with more complicated splicing might produce novel isoforms

119 more frequently. The splicing type that produced the novel transcripts - intron retention,

120 exon skipping, etc.- was complex: because the majority of transgressive genes were

121 alternatively spliced in the parents, the method of splicing that produced the novel

122 transcripts must be characterized in relation to multiple alternative splice forms.

123 Furthermore, comparisons between a transgressive isoform and an individual parent

124 isoform usually involved more than one type of splicing. For example, intron retention

125 and exon skipping sometimes occurred at different parts of the transcript

126 simultaneously. To summarize, intron retention in the transgressive isoforms was the

127 most common splicing type: $46 \%$ of comparisons between transgressive isoforms and

128 corresponding parent isoforms involved this splice type. Other splice types included

129 intron retention in the parent isoform (31\%; percentages not exclusive), exon skipping in

130 the parent isoform (42\%), exon skipping in the transgressive isoform (37\%), alternative

131 splice site causing longer transgressive transcript (21\%), and alternative splice site

132 causing longer parent transcript (20\%). Splice types are listed in Table 1. 
133 Genes with transgressive splicing patterns were distributed across all 17 chromosomes.

134 However, the number of transgressive isoforms spliced per gene was sometimes

135 greater than one: three genes each had two novel isoforms, and a single gene had

136 three novel isoforms. The majority of transgressive genes had similar splicing patterns

137 between wild and domesticated parents, although $25 \%$ were differentiated between

138 parent taxa. In Smith et al. (2018), splicing had diverged in $40 \%$ of analyzed genes. This

139 indicates that divergence in splicing of a particular gene is not a prerequisite for the

140 production of novel isoforms.

142 Functional annotation of novel transcripts

143 Forty transgressive isoforms were homologous with 35 different Arabidopsis proteins

144 with different functional annotations (Table 1). No two transgressive genes had the

145 same Arabidopsis homolog; however, for each gene with multiple transgressive

146 isoforms, the isoforms aligned to different splice forms of the same protein in a cleanly

147 delineated manner. The annotations spanned a diverse array of functions, and no

148 functional category appeared predominant. There were five novel isoforms that did not

149 align well to any Arabidopsis proteins, two of which had a parent isoform with a

150 homolog. The annotations for these were translation initiation factor 3 subunit I and

151 hypothetical protein.

152 Most transgressive isoforms aligned to the same protein splice variant as one of the

153 corresponding parent isoforms. This result is consistent with the transgressive isoforms

154 being previously uncharacterized, although it is reasonable to suspect that this pattern 
155 is due to limitations of the alignment algorithm, or a less than comprehensive protein

156 database. Only two novel isoforms aligned to different proteins than the parent isoform.

157 In one of these cases, the transgressive isoform aligned to a different protein splice

158 variant than the parent isoform, but they had the same annotation: uncharacterized

159 protein family UPF0016, a suspected calcium transporter (Demaegd et al., 2014). In the

160 other case, the transgressive isoform aligned to a protein with a different accession than

161 the parent isoform, but only a subtly different functional annotation. The parent isoform

162 was annotated as an F-box/RNI-like superfamily protein, while the transgressive isoform

163 was most similar to the RNI-like superfamily protein. The transgressive isoform homolog

164 is annotated as being involved in the degradation of KRP1/ICK1, which is not a function

165 of the parent variant.

167 Open reading frame analysis

168 The longest open reading frame (ORF) had been altered in most novel transcripts.

169 Specifically, 31 novel isoform ORFs were different versions of the corresponding parent

170 ORF, or different than all parent ORFs if the parents spliced multiple isoforms. Twenty-

171 two were shorter than the most similar parent ORF, eight were longer, and one novel

172 ORF was the same length as the parent version but differed in a six amino acid

173 sequence on the 5 ' end. Twenty-five of the transgressive ORFs differed by $>25$ amino

174 acids compared to the most similar parent ORF. In 13 novel splicing cases, an intact

175 parent ORF was present in the transgressive isoform; in these examples, we assumed

176 that the overall function of the gene was unaffected. In the remaining transgressive 
177 gene, also the shortest transgressive gene identified, no ORF was identified in the

178 parent isoform or the novel isoform.

181 We evaluated potential relationships between transgressive splicing and 15

182 measurements related to hybrid stress response. The measurements included: (i, ii) the

183 number of days until wilting and the number of days until death after watering ceased;

184 (iii, iv) soil water content at two timepoints; and ( $\mathrm{v}-\mathrm{xv})$ a set of correlated

185 measurements related to plant size including: height at three timepoints, dry and wet

186 weight, longest leaf length and width at two time points, and most recent fully expanded

187 leaf length and width. The transgressive isoform proportions were scaled and averaged

188 to obtain a cumulative transgressive isoform value for each RIL. Three of the size-

189 related traits had relationships with transgressive splicing: most recent fully expanded

190 leaf width ( $p=0.01$; Fig. 2A), dry weight $(p<0.01$; Fig. 2B), and wet weight $(p=0.02$;

$\left.191 r^{2}=0.06\right)$. This analysis predicted the most transgressive seedlings to have $47 \%$ less

192 mass (dry weight) compared to the least transgressive seedlings, although

193 transgressiveness only partly explained variation in plant size. 

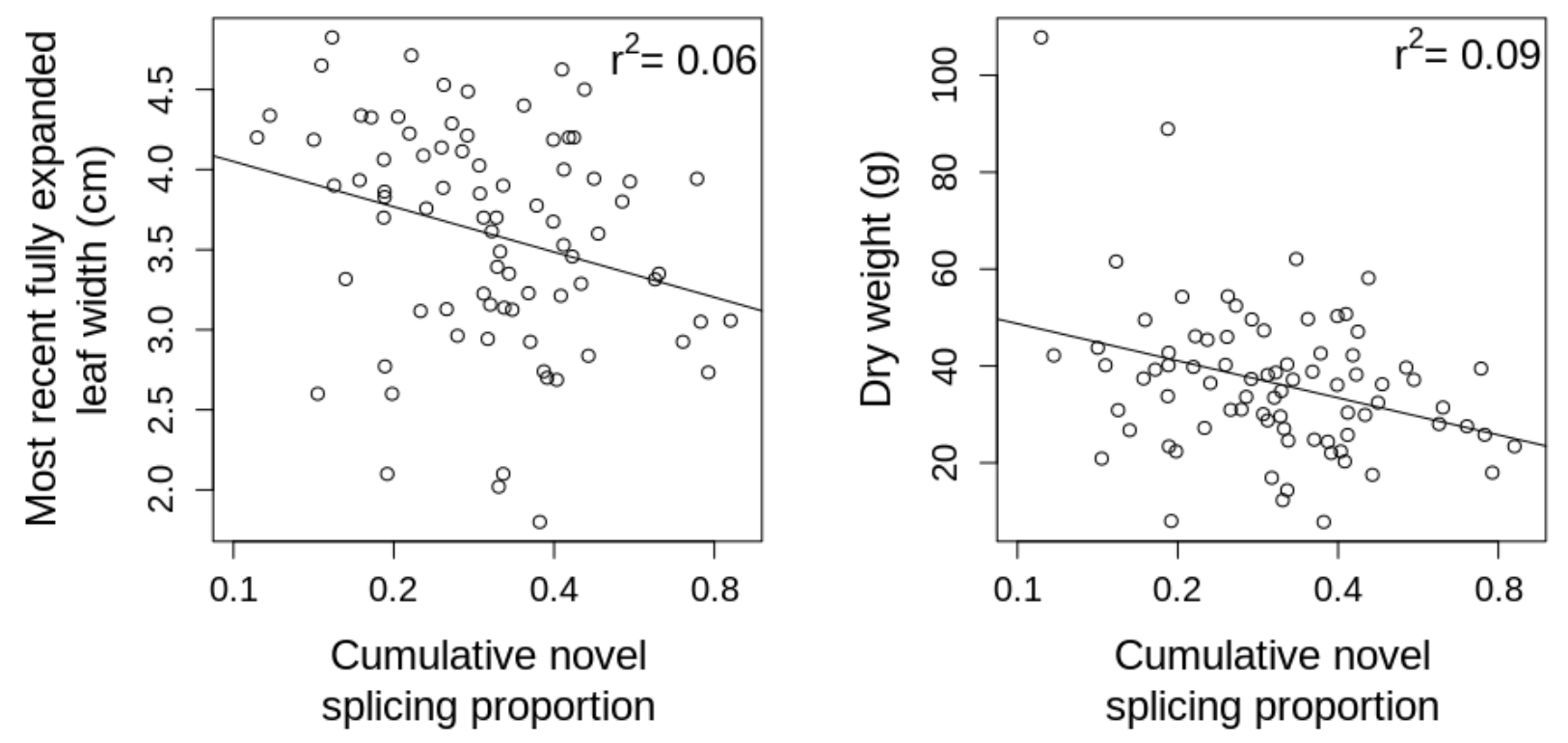

Figure 2. Stress response phenotypes versus transgressive splicing proportion. The $x$-axis is stretched to show log scale.

197 Additionally, we applied a principal component analysis to the eleven size traits, and the

198 first principle component (88\% of variance explained) was correlated with cumulative

199 transgressive splicing $\left(p=0.01 ; r^{2}=0.06\right)$. No individual isoform splicing levels had

200 significant correlations with plant size or other measurements after correcting for

201 multiple tests. Genome wide heterozygosity was not significantly correlated with plant

202 size or other measurements.

204 Overdominant regulatory loci were associated with novel splicing

205 To explore the genetic basis of each transgressive isoform, we scanned the genome for

206 quantitative trait loci (QTLs) that had statistical associations with transgressive splicing.

207 We detected QTLs for 19 different novel isoforms; these QTLs may regulate novel 
208 isoform splicing (Fig. 3, top row; Table 1). Most QTLs were associated with a single

209 transgressive splice form, but seven QTLs each regulated two to five novel isoforms.

210 The variance explained by each QTL ranged from $13 \%$ to $95 \%$ (median $=37 \%$ ). The

211 genomic position for most QTLs (85.7\%) was more than 5cM away from the gene with

212 transgressive splicing.
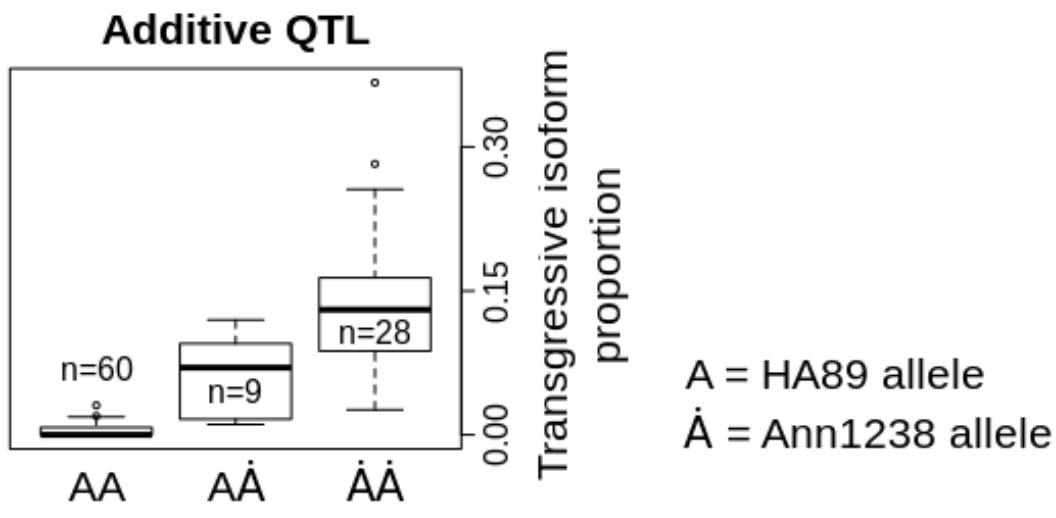

Epistatic QTLs

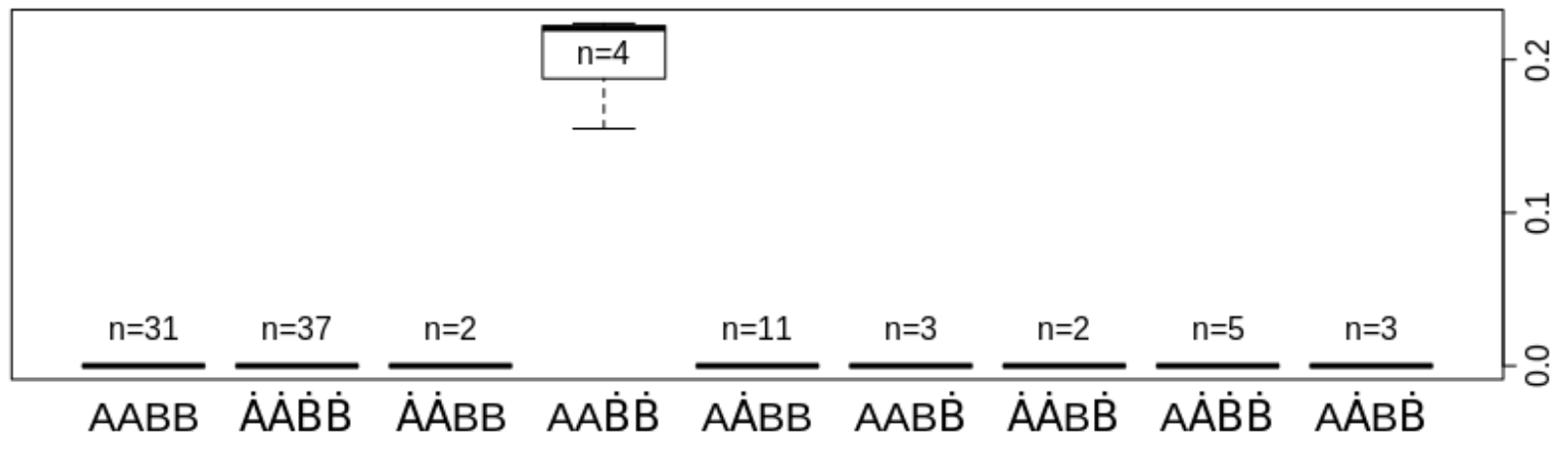

Figure 3. Transgressive splicing proportion for each genotype at example QTLs. (top left)

215 Proportion of novel isoform \#31 at an additive QTL (Chr. 11, $47.79 \mathrm{cM}$ ). (top right) Proportion of 216 novel isoform \#38 at an overdominant QTL (Chr. 13, $71.54 \mathrm{cM})$. (bottom) Proportion of novel isoform \#2 for a pair of epistatic QTLs (Chr. 13, 70.78 cM; Chr. 16, $3.47 \mathrm{cM})$.

218 For each of the identified QTLs, we examined whether the heterozygous genotype was

219 associated with transgressive splicing. Although the mapping population was inbred, the 
220 RILs retained heterozygosity at $10 \%$ of genomic sites on average. Eleven novel

221 isoforms had QTLs with overdominance effects: individuals that were heterozygous at

222 the QTL had a higher proportion of the transgressive isoform than either homozygous

223 genotype group (Fig. 3, top right; Table 1). This indicates that alleles from each parent

224 interacted non-additively to produce the transgressive splice type.

225 For only one novel splice form, the genotype at the QTL associated with transgressive

226 splicing was homozygous Ha89. The homozygous Ann1238 genotype was associated

227 with transgression for all remaining QTLs. Although fourteen novel isoforms were

228 regulated by more than one QTL, in these instances each individual QTL-effect came

229 from the same parent allele or overdominance. Therefore, the identified QTLs did not

230 show evidence for complementary gene action, where alleles from both parents have

231 effects that combine additively to produce the transgressive isoform.

233 Epistasis underlies transgressive splicing

234 Next, we explored whether alleles at different loci interacted non-additively to produce

235 the transgressive splice type. Irrespective of the single-QTL scan (above) we scanned

236 the genome for pairs of loci where the effect of one locus depended on the genotype at

237 the other locus. Sixteen novel splice forms could be explained by interacting regulatory

238 loci $\left(2.2 \times 10^{-20}<\mathrm{p}<3.1 \times 10^{-11}\right.$; Fig. 3, bottom panel; Table 1). The number of

239 epistatic QTLs per novel isoform ranged from 1 to 9 (median $=1.5$ ). Most epistatic

240 QTLs were trans, although $11.4 \%$ of interactions involved a QTL within 5cM of the gene

241 with transgressive splicing. 
242 It was revealing that significant epistasis was detected for seven novel isoforms with

243 QTLs identified in the initial single-locus scan. Three novel isoforms had single-QTLs

244 with genomic positions that overlapped with an epistatic QTL. This suggests that some

245 of the single-QTLs may have been initially detected because of epistatic interactions

246 with other loci. As a final check, we inspected whether the previously identified single-

247 QTLs interacted with each other. Of the 14 novel isoforms that were each regulated by

248 two or more single-QTLs, there were two pairs of interacting QTLs that affected the

249 same novel isoform $\left(1.4 \times 10^{-8}<p<3.3 \times 10^{-6}\right)$. Nine additional QTLs pairs

250 affecting eight novel isoforms showed evidence of weak epistasis $\left(1.1 \times 10^{-3}<p<\right.$

$\left.2513.8 \times 10^{-2}\right)$.

254 The spliceosome is a protein and snRNA complex that is responsible for the removal of

255 introns and splicing-together of exons during alternative splicing. Using genomic

256 resources for Arabidopsis to identify spliceosome genes in sunflower, we examined

257 which individual spliceosome components were involved in transgressive splicing. We

258 found associations between the expression level of individual spliceosome components

259 and splicing of 16 different transgressive isoforms (Fig. 4; Table 1). While each novel

260 isoform usually had only one spliceosome component association, five novel isoforms

261 each had more than one association, and four spliceosome components were each

262 used by more than one novel isoform. For example, the expression of U1 spliceosomal 
263 RNA was positively correlated with splicing of three transgressive splice forms. Our

264 novel isoforms were not spliceosome RNA or protein components, themselves.

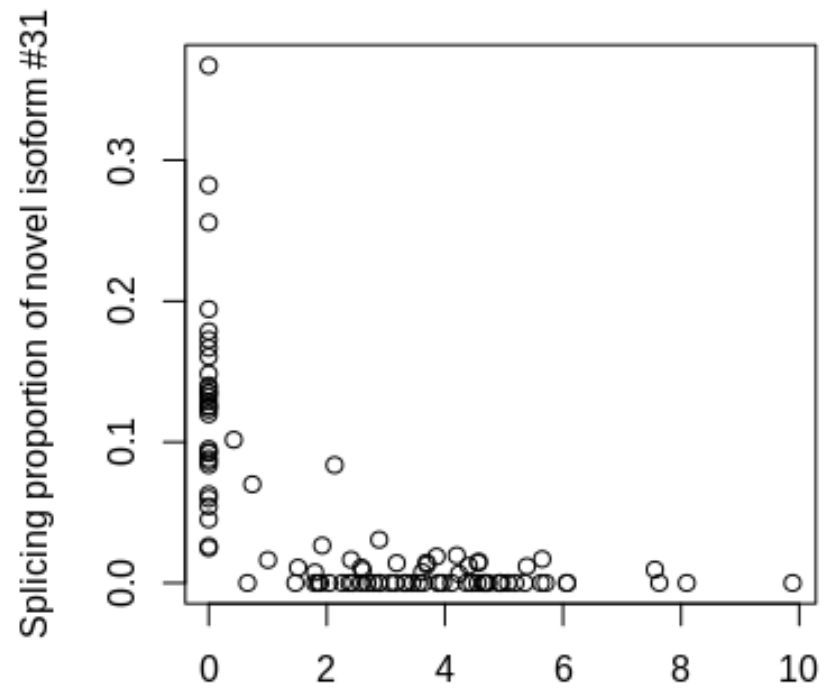

Figure 4. Novel isoform splicing versus spliceosome component expression.

267 We investigated which type of regulatory factor, QTLs or spliceosome component

268 expression, was more important in the splicing of novel isoforms. There were 15 novel

269 splicing examples where the transgressive isoform was associated with both (i) at least

270 one identified regulatory QTL and (ii) at least one spliceosome component. Using a

271 linear model with individual variables for each spliceosome component and QTL

272 associated with a novel isoform, we partitioned the sum of squares explained by each

273 factor. For six novel isoforms, the expression of the spliceosome components explained

274 more variance in splicing than the QTLs. This pattern of variance propagation suggests

275 that some- but not all- spliceosome components were more closely involved in novel

276 isoform production. The QTLs in these six cases affected novel splicing less directly,

277 perhaps through regulation of the spliceosome. 
278 Next, we inspected whether the QTLs primarily regulated spliceosome components

279 instead of directly regulating novel splicing itself. This was achieved by regressing each

280 QTL against each spliceosome component, and against novel isoform splicing. There

281 were eleven QTLs that explained more variance in the expression of a spliceosome

282 component than the splicing of the novel isoform. These QTLs were associated with

283 four different novel isoforms.

284 Last, we found statistical interactions between spliceosome components and QTLs

285 regulating the same novel isoform in eight novel splicing cases $\left(3.2 \times 10^{-35}<p<0.03\right.$

286 ), indicating that the two effects were sometimes interdependent. Looking back to the

287 parental samples, two of the spliceosome components that interacted with QTLs had

288 differentiated gene expression between parental genotypes $\left(1.3 \times 10^{-3}<p<8.8 \times\right.$

$28910^{-3}$ ). The latter signals that the parental genotypes have heritable variation in

290 spliceosome expression that could lead to genetic incompatibilities in the hybrids.

\section{Discussion}

\section{The evolution of new exons}

294 Much of what we know about alternative splicing evolution describes changes that

295 occurred over large evolutionary timescales, for example rates of intron gain and loss

296 and differences in intron numbers among widely divergent taxa (Lynch and Walsh,

297 2007). The analysis presented here shows that RNA splice sites can evolve in a small

298 number of generations through rewiring of existing regulatory networks. This contrasts 
299 with an existing model for splice site evolution predicting that mutations in the spliced

300 gene sequence itself would be the main source of new exons (Keren et al., 2010).

301 Another interesting pattern is that most of the affected genes had relatively high isoform

302 diversity and complex splicing. This suggests that genes with higher splicing complexity

303 may have a higher probability of creating new splice forms.

304 Four genes with novel splicing (novel isoforms 16, 23, 32, 33) each had a single mRNA

305 form in the parental samples. These parental transcripts each aligned to the reference

306 DNA in multiple pieces, indicating that they were spliced constitutively. These cases are

307 of particular interest because they might tell us something about how alternative splicing

308 evolved from constitutive splicing (assuming that genes with introns were an

309 intermediate evolutionary state; Ast 2004). These four novel isoforms were usually

310 spliced at low levels in the hybrids, but one isoform was the predominant splice type in

311 the hybrid samples. For three of these genes, the new alternative splice form retained

312 an intron, which is consistent with intron retention being the most common splice type in

313 plants (Chamala et al., 2015). In the fourth gene, the alternative splice type was a

314 combination of a newly incorporated exon and an alternative splice site. The latter case

315 fits a model where a new exon originates and then may evolve to become more

316 frequent. The gene sequence being spliced did not appear to be mutated in examined

317 samples, which instead suggests a regulatory change. Indeed, we found QTLs

318 associated with three of the above novel splice forms.

319 The longest ORF was inferred to be compromised in half of the novel isoforms in the

320 current study, by frameshifts and truncations. We assume that frameshifts in mRNAs

321 are costly for the organism, for example by affecting protein stability or binding domains, 
322 or by causing more serious disorders; although, nonsense-mediated decay may reduce

323 some of these effects. On the other hand, new splice sites may occasionally lead to

324 novel protein functionality, which could be beneficial if combined with the right adaptive

325 landscape. Most of the novel isoforms in the current study had the same closest

326 Arabidopsis homolog as the corresponding parental splice form. Therefore, the function

327 of the novel isoforms may be conserved, or the function may be altered but as of yet

328 uncharacterized. Experiments are needed to evaluate the function of the novel

329 transcripts. As described by Keren et al. (2010), in cases where the ancestral isoform is

330 still present, a non-coding mRNA or non-functional protein may have inherent regulatory

331 function through limiting the production of functional proteins from the same gene.

332 A novel transcript might be thought of as qualitatively different than the parental

333 transcript, especially if the corresponding protein function is altered. However, most

334 novel transcripts in our dataset were spliced at low levels and the parental isoform

335 remained the predominant form in the hybrids. Therefore, the presence of a new splice

336 site may represent only a gradual step in the transition in alternative splicing for a gene.

337 Simultaneously, recently evolved splice sites might endure a natural trial phase during

338 which selection decides whether or not to favor the new splice type. Through this

339 process, changes in splicing would evolve gradually, similar to other quantitative traits.

341 The genetic basis of novel splicing

342 Novel transcripts in the current study were found in hybrid samples and absent in the 343 parental samples, which is an extreme example of transgressive segregation. This type 
344 of inheritance indicates three possible genetic bases for the novel isoforms: epistasis,

345 overdominance, or complementary gene action; although complementary gene action is

346 generally accepted as the more common genetic basis for transgressive segregation

347 (deVicente and Tanksley, 1993; Rieseberg et al., 1999; Stelkens and Seehausen,

348 2009). In the current study, QTLs were detected for $64 \%$ of novel isoforms. We did not

349 experimentally verify any genetic associations.

350 Epistatic interactions explained $36 \%$ of novel splicing cases. The Rieseberg et al.

351 (1999) review reported only one example of transgressive segregation caused by

352 epistasis, however many of the reviewed studies did not test for epistasis. Stelkens and

353 Seehausen (2009) hypothesized that epistasis and complementary gene action would

354 explain most transgressive traits. This genetic basis for transgressive splicing is

355 intuitive, because alternative splicing ordinarily relies on epistatic gene networks for the

356 assembly and regulation of the spliceosome. In fact, we found that the expression levels

357 of particular spliceosome proteins and spliceosome RNAs were correlated with the

358 production of novel isoforms. In a subset of cases, the detected QTLs were more tightly

359 associated with the expression of certain spliceosome components than the novel

360 isoform itself, suggesting that the QTLs may regulate expression- or splicing- of the

361 spliceosome. Furthermore, QTLs often had nonadditive interactions with spliceosome

362 components, some of which had divergent gene expression in the parent taxa.

363 Biologically, interactions involving the spliceosome may represent a specific type of

364 hybrid incompatibility that leads to aberrant splicing.

365 In our dataset, inbreeding left relatively little heterozygosity in the RILs; nonetheless, we

366 found that $24 \%$ of novel splice forms could be explained by overdominance at one or 
367 more QTLs. Overdominance, here, does not suggest that the hybrid phenotype is

368 superior or better adapted. Few other transgressive phenotypes were explained by

369 overdominance in the Rieseberg et al. (1999) review, but Stelkens and Seehausen

370 (2009) reported many examples of heterosis in F1 hybrids. We would expect novel

371 isoforms caused by overdominance to be even more common in natural hybrid zones

372 due to higher heterozygosity. Transgressive phenotypes caused by overdominance are

373 ephemeral, except in the case of allopolyploidy, because the heterozygous genotype

374 cannot become fixed.

375 We did not find evidence for complementary gene action, where alleles from both

376 parents have effects that add together to cause transgression. Although, it is possible

377 that many undetected loci with small effects could contribute to transgressive splicing.

378 Unexpectedly, individual QTLs were identified for six novel isoforms that could not be

379 explained by overdominance or interactions with other loci. This result highlights a

380 limitation in our ability to detect regulatory loci, because otherwise we would expect to

381 see the aforementioned transgressive isoforms in one of the parental lines. It was

382 illuminating that some of the QTLs detected in the single-locus scan had significant

383 interactions with other regulatory loci; the remaining single-QTLs may have interacted

384 with the genomic background more subtly, involving many small interaction effects. In

385 fact, marginal epistasis was detected for three additional novel isoforms $(p<0.1$

386 before correcting for multiple tests). Alternatively, a higher-order interaction scan might

387 be required to see some kinds of epistasis. We did not find interactions or other QTLs

388 involving cpDNA. 
391 Following a period of allopatry, mutations that were benign in one parent genetic

392 background may be incompatible with the opposite parent genome. The field of

393 speciation has produced a wealth of theory regarding this type of postzygotic

394 incompatibility (Orr 1997; Orr and Turelli 2001; Turelli et al., 2001; Coyne and Orr

395 2004). Although BDM incompatibilities have maintained a central position in speciation

396 theory, empirical evidence for this type of reproductive isolation has only begun to

397 accumulate in recent decades (e.g. Brideau et al., 2006; Cattani and Presgraves, 2009;

398 Anderson et al., 2010; Matute et al., 2010; Moyle et al., 2010). Empirical evidence for

399 BDM incompatibilities is infrequent partly because sterile hybrid lineages are difficult to

400 study and epistasis is difficult to detect. We still don't fully understand how common

401 BDM incompatibilities are, what types of genes are involved, and how important they

402 are to allopatric speciation.

403 A breakdown in splicing regulation that leads to frameshifts in mRNA transcripts may be

404 considered an incompatibility if it contributes to reproductive isolation. It is not

405 uncommon for abnormal splicing of a single gene to be harmful: $10-15 \%$ of mutations

406 underlying human diseases affect splicing (Nissim-Rafinia and Kerem, 2002). Although

407 reproductive isolation was not directly tested in the current study, splicing malfunction

408 led to mRNA frameshifts, and disrupted splicing was negatively correlated with seedling

409 growth rate. We point out the feasibility of a scenario where an epistatic interaction

410 involving the spliceosome simultaneously affects splicing of many loci and could lead to

411 reduced hybrid fitness. Other links have been found between alternative splicing and

412 adaptive radiation: Terai et al. (2003) showed that the putative pigmentation gene 
413 hagoromo has increased splicing diversity in cichlid species, in particular ones that have

414 recently radiated, and different species exhibited fixed splicing differences. Also in

415 cichlids, Singh et al. (2017) showed that alternative splicing patterns had diverged more

416 dramatically between species than gene expression levels, and genes with

417 differentiated splicing were associated with jaw specialization. Last, the noteworthy

418 Agouti gene produced multiple isoforms in the skin of deer mice, and one particular

419 isoform was associated with light-sand camouflage (Mallarino et al. 2017).

420 Future research is warranted to investigate splicing malfunctions in other hybridizing

421 taxa, particularly between wild populations or species. Speciation researchers often test

422 for epistasis associated with a particular reproductive isolation phenotype (e.g. sterility).

423 If disrupted splicing is common in other taxa, researchers will have access to many

424 potential targets for studying negative epistatic effects. Also, expression QTLs tend to

425 have a high signal to noise ratio, which may improve detection of regulatory loci for

426 splicing phenotypes. Most transgressive splicing cases in our study involved genes

427 where splicing had not differentiated between parental lineages. This is consistent with

428 the findings of Stelkens and Seehausen (2009), who hypothesized that stabilizing

429 selection- not divergent selection- would favor transgressive phenotypes; however, that

430 hypothesis was based on a model of complementary gene action, which we did not find

431 evidence for. Contrasting predictions have been made about the relationship between

432 genetic divergence and the frequency of transgression (Rieseberg et al., 1999; Stelkens

433 and Seehausen., 2009). Additional research is necessary to determine what factors

434 predict transgressive splicing. 
437 Plant material and RNA Sequencing.

438 The raw sequence data from Smith et al. (2018) were reanalyzed in the current study.

439 Ha89 is an inbred line commonly used in research and breeding (US Department of

440 Agriculture Ames 3963). Ann1238 was derived from material collected at Cedar Point

441 Biological Station, Keith County, Nebraska. Three seedlings from each parental

442 accession and each of 100 sixth-generation recombinant inbred lines were grown in

443 greenhouse conditions to address stochastic variation in expression. Above-ground

444 tissue was frozen in liquid nitrogen, and total RNA was extracted following standard

445 protocols. Nonnormalized Illumina RNA-sequencing (RNA-seq) libraries were

446 sequenced on a HiSEq2000 system.

449 We built a de novo transcriptome assembly including all parental samples and RILs

450 using the program Trinity (v. 2.8.1) with default settings. This assembly differed from the

451 Trinity assembly from Smith et al. (2018), because the previous assembly included only

452 the parental samples. Henceforth, all data are presented for the first time. Custom

453 scripts were used for all of the following steps and analyses, except when existing

454 software is explicitly named. All custom code is available at

455 https://github.com/chriscrsmith/SunflowerTransgressiveSplicing. 
456 After completion of the assembly, the following initial steps were used to verify the

457 assembled transcripts. All transcripts were aligned to the Ha412-HO genome (Staton

458 and Rieseberg, 2019; Todesco et al., 2020), a domesticated sunflower genotype, using

459 BLAST (McGinnis and Madden, 2004). Blast hits with percent identity below 95\% were

460 ignored. We required the entire transcript to have aligned ambiguously to the same

461 genomic region, but with up to $10 \%$ of the transcript length missing from the ends.

462 Additionally, the genomic alignment was required to be at least $50 \%$ single-copy- we

463 delineated single copy sites by aligning whole genome sequencing data to the Ha412-

$464 \mathrm{HO}$ reference and visualizing the distribution of read depths per site (S1 Text). If

465 isoforms that were assigned the same gene-ID by Trinity aligned to different genomic

466 locations, then we considered them as having been transcribed from different genes for

467 all subsequent analyses.

468 Next, we used the following procedure to distinguish alternative splice forms assigned

469 by Trinity from alternative alleles. We created a multiple sequence alignment of all

470 isoforms from a gene using MUSCLE (v. 3.8.31), or, if only two isoforms, a pairwise

471 sequence alignment using the EMBOSS (v. 6.6.0.0) needle program. For each pair of

472 transcripts in the alignment: if at least one insertion/deletion or substitution larger than

473 our minimum exon size cutoff- 25 bp- was present, we considered the transcripts to be

474 legitimate alternative splice forms. If any shorter runs of differences, e.g. a single

475 nucleotide polymorphism (SNP), were present, we considered the transcripts to be

476 alternative alleles of the same splice form; except, if both a $>25 \mathrm{bp}$ run and a shorter

477 run were present, we deemed the relationship ambiguous. 
478 We then applied the following filters to identify transgressive isoforms. For each

479 potential transgressive isoform, we required: (i) all parents to have zero reads of the

480 transgressive isoform and any alternative alleles, (ii) at least one hybrid to have

481 substantial expression- > 1 TPM- of the transgressive isoform, and for there to be (iii) at

482 least one alternative splice form with > 1 TPM in every parent. Because these isoforms

483 are expressed at a substantial level, they are presumably distinct from stochastic

484 splicing errors or noise.

Testing for splicing differentiation

487 To test for divergent splicing composition between wild and domesticated sunflower 488 genotypes, we first applied an isometric log ratio transformation to the proportions of 489 each isoform (Smith et al., 2018). Then we used a t-test, or a MANOVA in the case of

490 more than two parent isoforms, to test for a difference in splicing composition.

492 Gene annotation

493 Transcripts were aligned to the Araport11 table from The Arabidopsis Information

494 Resource (Berardini et al. 2015) using BLASTX (McGinnis and Madden, 2004) with e495 value $<10^{-20}$ cutoff. The best hit was retained for each transcript. 
498 The program ORFfinder (National Center for Biotechnology Institute website;

499 www.ncbi.nlm.nih.gov/orffinder/) with default settings was used to identify open reading

500 frames for each novel isoform and all parent isoforms corresponding to the same gene.

Ha89 × Ann1238 RIL seedlings, and inbred parental lines, were grown in a common

504 environment in greenhouses at the University of British Columbia in the spring of 2011, 505 with plants watered and fertilized daily. Plant height, leaf lengths and widths, and other 506 measurements were taken at 4 weeks and 6 weeks after germination. At 6 weeks, 507 watering was stopped entirely, with daily observations checking for wilting and death of 508 each plant. Soil moisture content was measured with a HydroSense CS620 soil 509 moisture system (Campbell Scientific) at 6 days after the initiation of the drought and on 510 the day of first observed wilting for each plant. Plant measurements from this 511 experiment are in Table S1.

\section{Single nucleotide variant identification and filtering}

514 SNPs were identified by aligning RNA-seq reads to the Ha412-HO transcriptome

515 (Renaut et al., 2013) by using bwa mem v0.7.15. SNPs were called by using SAMtools

516 v1.4.1. We filtered SNPs that aligned to non-single-copy genomic regions (S1 Text) and

517 marked genotypes with fewer than ten read depth as missing data. After the initial

518 filters, we kept 66,893 SNPs that showed fixed differences between wild and 
519 domesticated parental samples. Next, we obtained genetic map positions for each fixed

520 parent SNP by aligning transcriptome contigs to the Ha412-HO genome using BLAST

521 (e value $\leq 10^{-20}$; ID $\geq 90 \%$ ) and linearly interpolating $\mathrm{cM}$ positions from the $\mathrm{Ha} 412-\mathrm{HO}$

522 genetic map (Staton and Rieseberg, 2019; Todesco et al., 2020). Markers that aligned

523 well were examined in the RILs, and genotypes with fewer than ten reads were marked

524 as missing. Markers were excluded if present in fewer than $35 \%$ of RILs, resulting in

52513,874 filtered SNPs. Last, we applied a conservative imputation step to the filtered

526 SNPs: if a marker with missing data occurred between two markers with the same

527 genotype that were within $10 \mathrm{cM}$, we assigned the same genotype to the missing

528 marker. Otherwise, missing genotypes were left as missing. This step filled in $31 \%$ of

529 missing data, and $1.4 \%$ of genotypes overall.

QTL mapping

532 To explore the genetic basis of each transgressive isoform, we used the program R/qtl

533 (Broman et al., 2003) to conduct standard interval mapping with a non-parametric

534 model. The mapped phenotype value was the proportion of transgressive isoform

535 spliced relative to overall gene expression. The densest genetic map grid $(s t e p=0)$ was

536 used, and the genotyping error rate was set to zero (error.prob $=0$ ) because the

537 genotypes were already carefully filtered (above). We generated a null distribution of

538 LOD scores for each novel isoform being mapping by permuting the phenotypic

539 measurements relative to the genotypes: for 10,000 iterations, the splicing

540 measurements were shuffled before applying the QTL scan, and the largest LOD score 
541 was recorded from each permutation; the $95^{\text {th }}$ percentile of this distribution was used as

542 the significance threshold (Broman and Sen, 2009). We allowed a single QTL per

543 chromosome and assigned the QTL peak as the marker position with the highest LOD

544 score. We delineated the QTL region as the range of SNP markers with LOD scores

545 within $25 \%$ of the peak. Overlapping QTL regions from different novel isoforms were

546 consolidated for reporting the total number of QTLs.

547 For each QTL identified in the above scan, we calculated the degree of dominance at

548 the QTL peak. The degree of dominance is the ratio of the dominance effect to the

549 additive effect, the former being the difference between the mean phenotype of the

550 heterozygote group and the midpoint between the mean phenotypes of each

551 homozygote group, and the latter being half the difference between the mean

552 phenotypes of the two homozygous groups (Kenney-Hunt, 2006; Ishikawa, 2009). A

553 degree of dominance equal to zero means perfect codominance, 0 to 1.5 means one

554 allele is dominant, and greater than 1.5 was interpreted as overdominance.

557 For each novel isoform, we scanned the genome for pairs of loci where alleles from 558 each parent were interacting to cause transgressive isoform splicing. The proportion of

559 transgressive isoform spliced relative to overall gene expression was used as the 560 phenotype value, and if an individual had insufficient expression ( $<1$ TPM) at the 561 gene-level they were assigned missing data. We limited the search to pairs of loci on 562 different chromosomes that met the following criteria: (i) at least three individuals were 
563 represented that had non-missing phenotype data and were homozygous for the Ha89

564 allele at both loci- notated $A A B B-$, (ii) at least three individuals were represented that

565 were homozygous for the Ann1238 allele at both loci- $\dot{A} \dot{A} \dot{B} \dot{B}-$, (iii) at least three

566 individuals were represented for at least one mixed genotype - $\dot{A} \dot{A} B B, A A \dot{B} \dot{B}, A \dot{A} B B$,

$567 A A B \dot{B}, \dot{A} \dot{A} B \dot{B}, A \dot{A} \dot{B} \dot{B}$, or $A \dot{A} B \dot{B}$. Encoding the genotypes as allele dosages- 0 , 1 , or $2-$,

568 we tested for an interaction effect between markers using a multiple linear regression.

569 We used a Bonferroni correction to obtain a significance threshold for the epistasis

570 scan: the total number of tests for all 45 phenotypes was $1.61 \times 10^{9}$, and the

571 significance threshold became $p<3.11 \times 10^{-11}$. Last, we applied the following post

572 hoc filter to avoid outlier effects: if the most transgressive genotype group had fewer

573 than three individuals represented, we counted an otherwise significant test as non-

574 significant. We allowed a single QTL pair for each pair of chromosomes and assigned

575 the QTL peak as the marker position with the smallest $p$ value. We delineated the QTL

576 region as the range of SNP markers with p-values within $25 \%$ of the peak in log-space:

$577 \quad p<p_{\min }{ }^{(1-0.25)}$.

578 Interacting QTLs were reported from the above scan only. We conducted a similar

579 epistasis scan using R/qtl with standard two-locus interval mapping, the densest genetic

580 map grid (step $=0)$, and ignoring same-chromosome comparisons (

581 clean.output $=T R U E ;$ clean.distance $=999)$. One thousand permutations were used to

582 obtain a null distribution for the interaction LOD score, and the $95^{\text {th }}$ percentile was used

583 as the significance threshold. This scan found a total of 177 pairs of interacting QTLs for

58421 novel isoforms; however, all of these QTL-pairs were suspect. For a large proportion

585 of cases, it was clear that outliers had inflated statistical significance because the 
586 genotype group with the largest novel splicing proportion was represented by only one

587 sample. This issue is likely specific to our dataset: with $10 \%$ of markers heterozygous in

588 the mapping population, the two-locus genotypes that included heterozygotes $(A A B \dot{B}$,

$589 A \dot{A} B B, \dot{A} \dot{A} B \dot{B}, A \dot{A} \dot{B} \dot{B}$, and $A \dot{A} B \dot{B})$ were usually represented by at most one sample. For

590 the subset of QTL-pairs that did have sufficient sample sizes in each genotype group,

591 the detected interaction appeared to be an artifact of missing data imputation (interval

592 mapping involves the calculation of genotype probabilities for missing genotypes). Each

593 of these QTLs had many genotypes missing; for some of the identified QTLs, all

594 transgressive samples had missing data at both loci. We concluded that this method

595 was inappropriate given the particular attributes of the dataset.

Associations with individual spliceosome components

Sequences for Arabidopsis thaliana spliceosome protein and RNA components were

599 obtained from KEGG and arabidopsis.org. Spliceosome sequences were aligned to our

600 sunflower transcriptome using TBLASTN (McGinnis and Madden, 2004), and the best

601 hit with e-value $<10^{-20}$ was retained for each component. The total number of tests

602 was 4,275. With a Bonferroni correction, the significance threshold for this analysis was

$603 p<1.16 \times 10^{-5}$.

604 When a novel isoform was associated with both a spliceosome component and a

605 regulatory QTL, we partitioned the variance explained using a linear model including all

606 spliceosome components and QTLs associated with the novel isoform as predictors.

607 For epistatic QTL-pairs, only the interaction term was included in the model. Interaction 
608 terms were included for each possible spliceosome component-QTL combination. We

609 partitioned the sum of squares explained using eta-squared with type three sum of

610 squares. To examine whether a QTL explains more variance in novel isoform splicing or

611 expression of spliceosome components, we used individual linear models including one

612 predictor and one response variable: the QTL was the predictor, and individual

613 spliceosome components or the novel isoform phenotype was the response.

616 This work utilized both the BioFrontiers Computing Core at the University of Colorado at

617 Boulder (UCB) supported by BioFrontiers IT, and the RMACC Summit Supercomputer

618 supported by the National Science Foundation (awards ACl-1532235 and ACl-

619 1532236), UCB, and Colorado State University.

622 Sequence data reported in this paper are available as part of the Sequence Read

623 Archive: Bioproject no. PRJNA417714.

626 This work was funded by Genome Canada and Genome BC (LSARP2014-223SUN).

627 Work on this project was also paid for by startup funds and donations to the lab of NCK. 


\section{Author contributions}

630 Conceptualization, original draft preparation, and review and editing were contributed by

631 CCRS, LHR, BSH, and NCK. Formal analysis was performed by CCRS and NCK.

\section{References}

634 Anderson JB, Funt J, Thompson DA, Prabhu S, Socha A, Sirjusingh C, Dettman JR, Parreiras L, Guttman DS, Regev A, Kohn LM. Determinants of divergent adaptation and Dobzhansky-Muller interaction in experimental yeast populations.

Ast G. How did alternative splicing evolve?. Nature Reviews Genetics. 2004 Oct;5(10):773-82.

640 Bateson W. Heredity and variation in modern lights. Darwin and modern science. 1909.

641 Berardini TZ, Reiser L, Li D, Mezheritsky Y, Muller R, Strait E, Huala E. The arabidopsis information resource: Making and mining the "gold standard" annotated reference plant genome. genesis 53.

644 Brideau NJ, Flores HA, Wang J, Maheshwari S, Wang XU, Barbash DA. Two Dobzhansky-Muller genes interact to cause hybrid lethality in Drosophila. science. 2006 Nov 24;314(5803):1292-5. 
647 Broman KW, Sen S. A Guide to QTL Mapping with R/qtl. New York: Springer; 2009 Apr 23.

649 Broman KW, Wu H, Sen Ś, Churchill GA. R/qtl: QTL mapping in experimental crosses. $650 \quad$ Bioinformatics. 2003 May 1;19(7):889-90.

651 Cattani MV, Presgraves DC. Genetics and lineage-specific evolution of a lethal hybrid incompatibility between Drosophila mauritiana and its sibling species. Genetics.

Chamala S, Feng G, Chavarro C, Barbazuk WB. Genome-wide identification of evolutionarily conserved alternative splicing events in flowering plants. Frontiers in bioengineering and biotechnology. 2015 Mar 26;3:33.

Cheng CY, Krishnakumar V, Chan AP, Thibaud-Nissen F, Schobel S, Town CD. Araport11: a complete reannotation of the Arabidopsis thaliana reference genome. The Plant Journal. 2017 Feb;89(4):789-804.

Coyne JA, Orr HA. Speciation (Sinauer, Sunderland, MA). family of putative calcium transporters. PloS one. 2014 Jun 23;9(6):e100851.

663 DeVicente MC, Tanksley SD. QTL analysis of transgressive segregation in an interspecific tomato cross. Genetics. 1993 Jun 1;134(2):585-96.

665 Dobzhansky TH. Studies on hybrid sterility. II. Localization of sterility factors in 
667 Huang W, Chen X, Guan Q, Zhong Z, Ma J, Yang B, Wang T, Zhu W, Tian J. Changes of alternative splicing in Arabidopsis thaliana grown under different $\mathrm{CO} 2$ concentrations. Gene. 2019 Mar 20;689:43-50.

Huang Y, Lack JB, Hoppel GT, Pool JE. Evolution of Gene Expression and Splicing in Parallel Cold-Adapted Fly Populations. bioRxiv. 2019 Jan 1:795716.

672 Ishikawa A. Mapping an overdominant quantitative trait locus for heterosis of body weight in mice. Journal of heredity. 2009 Jul 1;100(4):501-4. D, Norgard E, Perel C, Cheverud JM. Quantitative trait loci for body size components in mice. Mammalian genome. 2006 Jun 1;17(6):526-37.

Keren H, Lev-Maor G, Ast G. Alternative splicing and evolution: diversification, exon definition and function. Nature Reviews Genetics. 2010 May;11(5):345-55.

Khokhar W, Hassan MA, Reddy AS, Chaudhary S, Jabre I, Byrne L, Syed NH. Genome-wide identification of splicing quantitative trait loci (sQTL) in diverse ecotypes of Arabidopsis thaliana. Frontiers in plant science. 2019;10:1160. environments. Evolution. 1966 Sep 1:315-36. Aug;9(8):977. 
Mallarino R, Linden TA, Linnen CR, Hoekstra HE. The role of isoforms in the evolution of cryptic coloration in Peromyscus mice. Molecular Ecology. 2017

691 Jan;26(1):245-58.

Matute DR, Butler IA, Turissini DA, Coyne JA. A test of the snowball theory for the rate of evolution of hybrid incompatibilities. Science. 2010 Sep 17;329(5998):1518-21.

694 McGinnis S, Madden TL. BLAST: at the core of a powerful and diverse set of sequence analysis tools. Nucleic acids research. 2004 Jul 1;32(suppl_2):W20-5.

Moyle LC, Nakazato T. Hybrid incompatibility "snowballs" between Solanum species. Science. 2010 Sep 17;329(5998):1521-3.

Nissim-Rafinia M, Kerem B. Splicing regulation as a potential genetic modifier. TRENDS in Genetics. 2002 Mar 1;18(3):123-7. Nov;28(1):195-218.

Rieseberg LH, Archer MA, Wayne RK. Transgressive segregation, adaptation and speciation. Heredity. 1999 Oct;83(4):363-72.

Renaut S, Grassa CJ, Yeaman S, Moyers BT, Lai Z, Kane NC, Bowers JE, Burke JM, Rieseberg LH. Genomic islands of divergence are not affected by geography of speciation in sunflowers. Nature communications. 2013 May 7;4(1):1-8.

707 Scascitelli M, Cognet M, Adams KL. An interspecific plant hybrid shows novel changes 
710 Severing El, van Dijk AD, Stiekema WJ, van Ham RC. Comparative analysis indicates

711 that alternative splicing in plants has a limited role in functional expansion of the

712 proteome. BMC genomics. 2009 Dec 1;10(1):154.

713 Singh $\mathrm{P}$, Börger $\mathrm{C}$, More $\mathrm{H}$, Sturmbauer $\mathrm{C}$. The role of alternative splicing and

714 differential gene expression in cichlid adaptive radiation. Genome biology and

715 evolution. 2017 Oct;9(10):2764-81.

716 Slatkin M, Lande R. Segregation variance after hybridization of isolated populations.

717 Genetics Research. 1994 Aug;64(1):51-6.

718 Smith CC, Tittes S, Mendieta JP, Collier-zans E, Rowe HC, Rieseberg LH, Kane NC.

719 Genetics of alternative splicing evolution during sunflower domestication.

720 Proceedings of the National Academy of Sciences. 2018 Jun 26;115(26):6768-

$721 \quad 73$.

722 Staton, S. E. \& Rieseberg, L. H. Sunflower Genome Database,

723 https://www.sunflowergenome.org/ (2019).

724 Stelkens R, Seehausen O. Genetic distance between species predicts novel trait

725 expression in their hybrids. Evolution: International Journal of Organic Evolution.

2009 Apr;63(4):884-97.

727 Terai Y, Morikawa N, Kawakami K, Okada N. The complexity of alternative splicing of

728 hagoromo mRNAs is increased in an explosively speciated lineage in East

729 African cichlids. Proceedings of the National Academy of Sciences. 2003 Oct

28;100(22):12798-803. 
731 Thatcher SR, Zhou W, Leonard A, Wang BB, Beatty M, Zastrow-Hayes G, Zhao X,

732 Baumgarten A, Li B. Genome-wide analysis of alternative splicing in Zea mays:

733 landscape and genetic regulation. The Plant Cell. 2014 Sep 1;26(9):3472-87.

734 Todesco M, Owens GL, Bercovich N, Légaré JS, Soudi S, Burge DO, Huang K, Ostevik

735 KL, Drummond EB, Imerovski I, Lande K. Massive haplotypes underlie ecotypic

736 differentiation in sunflowers. Nature. 2020 Jul 8:1-6.

737 Turelli M, Barton NH, Coyne JA. Theory and speciation. Trends in ecology \& evolution.

2001 Jul 1;16(7):330-43.

739 Zhang $\mathrm{C}$, Yang H, Yang $\mathrm{H}$. Evolutionary character of alternative splicing in plants.

$740 \quad$ Bioinformatics and biology insights. 2015 Jan;9:BBI-S33716.

741 Zhang Z, Xiao B. Comparative alternative splicing analysis of two contrasting rice

742 cultivars under drought stress and association of differential splicing genes with

743 drought response QTLs. Euphytica. 2018 Apr 1;214(4):73. 